\title{
Sheathless Capillary Electrophoresis/Electrospray Mass Spectrometry Using a Carbon-Coated Fused-Silica Capillary
}

\author{
Yan Zin Chang and Guor Rong Her* \\ Department of Chemistry, National Taiwan University, Taipei, Taiwan, Republic of China
}

\begin{abstract}
A simple procedure was developed for preparing a carboncoated fused-silica capillary for use in sheathless capillary electrophoresis/ electrospray mass spectrometry (CE/ ESIMS). The tapered capillary tip was smeared with a marker pen before coating with carbon using a soft pencil. The layer from the ink of the marker pen was critical to the preparation of the carbon-coated capillary. The fabrication of a carbon-coated fused-silica capillary tip requires less than $1 \mathrm{~min}$. The stability of this carbon-coated fused-silica capillary is examined, and its utility in on-line sheathless CE/ESI-MS is demonstrated with the separation of berberine, coptisine, and palmatine chlorides. Although the carbon-coated fused-silica capillary tip is not as rugged as a gold-coated capillary, it is durable enough for sheathless CE/ESI-MS applications. Moreover, it is easy to refurbish the column once the performance of the tip is degraded.
\end{abstract}

Capillary electrophoresis (CE) is a powerful separation technique which has rapidly developed and matured since its introduction. ${ }^{1,2}$ Because of its salient universality, relatively high sensitivity, and structural elucidation capability, mass spectrometry is expected to become one of the most powerful and popular CE detectors. ${ }^{3,4}$ Currently, electrospray ionization (ESI) serves as the most common ionization method for CE/M S applications. ${ }^{5-7}$ One major requirement for using ESI-M S as an on-line detector in CE separations is the provision of electrical contact at the capillary outlet. The most widely used interface for commercial CE/ESIMS instrumentation is the sheath-flow interface. ${ }^{8} \mathrm{At}$ the sheathflow interface, a coaxial sheath liquid was introduced which served to establish the electrical contact for CE. This method offers several advantages, including reliability, simple fabrication, and

* Corresponding author. Tel: (886) 2-23690152, ext. 109. Fax: (886) 2-23638058. E-mail: grher@mail.ch.ntu.edu.tw.

(1) Jorgenson, J. W.; Lukacs, K. D. Anal. Chem. 1981, 53, 1298-1302.

(2) Jorgenson, J. W.; Lukacs, K. D. Science 1983, 222, 266-272.

(3) St. Claire, R. L. Anal. Chem. 1996, 68, 569R-586R.

(4) Burlingame, A. L.; Boyd, R. K.; Gaskell, S. J. Anal. Chem. 1996, 68, 599R$651 R$.

(5) Smith, R. D.; Wahl, J. H.; Goodlett, D. R.; Hofstadler, S. A. Anal. Chem. 1993, 65, 574A-584A.

(6) Cai, J.; Henion, J. J. Chromatogr., A 1995, 703, 667-692.

(7) Olivares, J. A.; N guyen, N. T.; Yonker, C. R.; Smith, R. D. Anal. Chem. $1987,59,1230-1232$.

(8) Udseth, H. R.; Barinaga, C. J .; Smith, R. D. Anal. Chem. 1988, 60, 19481952. easy implementation. One major drawback of the sheath liquid design is that the concentration of the analyte is substantially diluted due to the relatively high flow rate of sheath liquid.

Recently, the development of sheathless CE/ M S interfaces has attracted significant attention. 9,10 The coating of a conductive layer such as gold on the tip of the separation capillary column provides the electrical contact needed for both electrospray and CE separation. Among the sheathless CE/ M S techniques, the goldcoated fused-silica capillary method is the most popular. ${ }^{11-13}$ In general, the fabrication of gold-coated tapered capillaries requires special equipment and is also time consuming. ${ }^{14-16}$ In addition, the coated tips have a limited lifetime. The electrical contact is gradually degraded, and operation eventually becomes problematic, limiting the routine applications of CE/ ESI-MS.

One solution to this problem is the development of a disposable emitter. ${ }^{17,18}$ The use of disposable gold-coated tips butted to the CE column is an attractive approach to sheathless CE/ESI-M S, for it allows the original CE column to be retained. The performance of the butted tip interface was almost as good as that of the one-piece column, but less time consuming and resource consuming. However, it is important for the butted tip interface to ensure a good connection between the tip and the column. $M$ isalignment and/ or a bad connection will cause longer analysis times and/ or peak distortion.

Another solution to the problem is to develop an interface which is easy to make and also can be easily refurbished if the performance of the interface is degraded. In this paper, a simple method for making a carbon-coated tapered capillary column is described. The merits and limitations of this approach are discussed.

(9) Wahl, J. H.; Gale, D. C.; Smith, R. D. J. Chromatogr. 1994, 659, 217-222.

(10) Wahl, J. H.; Smith, R. D. J. Capillary Electrophor. 1994, 1, 62-71.

(11) Kriger, M. S.; Cook, K. D. Anal. Chem. 1995, 67, 385-389.

(12) Figeys, D.; Van Ostveen, I.; Ducret, A.; Aebersold, R. Anal. Chem. 1996, 68, 1822-1828.

(13) Kelly, J. F.; Ramaley, L.; Thibault, P. Anal. Chem. 1997, 69, 51-60.

(14) Severs, J. C.; Harms, A. C.; Smith, R. D. Rapid Commun. Mass Spectrom. 1996, 10, 175-1178.

(15) Severs, J. C.; Smith, R. D. Anal. Chem. 1997, 69, 2154-2158.

(16) Mazereeuw, M.; Hofte, A. J. P.; Tjaden, U. R.; Van Der Greefi, J. Rapid Commun. Mass Spectrom. 1997, 11, 981-986.

(17) Bateman, K. P.; White, R. L.; Thibault, P. Rapid Commun. M ass Spectrom. 1997, 11, 307-315.

(18) Bateman, K. P.; White, R. L.; Yaguchi, M .; Thibault, P. J. Chromatogr., A 1998, 794, 327-344.

10.1021/ac990535e CCC: $\$ 19.00$ C 2000 American Chemical Society Published on Web 12/16/1999 


\section{EXPERIMENTAL SECTION}

Reagents and Materials. Horse heart myoglobin, berberine chloride, and palmatine chloride were purchased from Sigma (St. Louis, M O). Coptisine chloride was obtained from Nacalai Tesque (Kyoto, Japan). M ethanol and 2-propanol of HPLC grade were purchased from J. T. Baker (Phillipsburg, NJ) and used without further purification. Deionized water (Milli-Q water system, Millipore Inc., Bedford, MA) was used in the preparation of the samples and buffer solution. All fused-silica capillaries were purchased from Polymicro Technologies (Phoenix, AZ). Pencils (Tombow Mono Inc.) and marker pens (Liberty Inc., Taipei, Taiwan) were used directly without any alteration.

The Carbon-Coated Tapered Capillary. The tapered capillary assembly utilized a $65 \mathrm{~cm} \times 75 \mu \mathrm{m}$ i.d. $\times 365 \mu \mathrm{m}$ o.d. fused-silica capillary column. The capillary tip was drawn manually using a vertically suspended section of capillary to which a small weight ( $30 \mathrm{~g}$, HPLC tee) had been attached. The capillary was slowly heated to the melting stage and then quickly withdrawn. The dimensions of the tip were about $50 \mu \mathrm{m}$ o.d. and $20 \mu \mathrm{m}$ i.d. and were controlled by removing the end of the tip with a ceramic cutter aided by visual inspection with a stereomicroscope. This tapered tip was further sharpened by etching it in $48 \% \mathrm{HF}$ for 15-30 min. During this process, the inside of the capillary was rinsed with deionized water $(2 \mu \mathrm{L} / \mathrm{min})$ to prevent widening of the tip. After etching, the capillary surface was rinsed with deionized water and purged with nitrogen to remove any traces of HF. The capillary tip was then smeared with a marker pen followed by carbon coating using a soft pencil (more details can be found in Results and Discussion).

Instrumentation. The sheathless CE/ ESI-M S instrumentation was configured in house. Briefly, the setup consisted of a $1000 R$ high-voltage power supply (Spellman, Plainview, NY) connected to a platinum electrode in a vial containing a CE buffer and operated in a constant-voltage mode. Separations were achieved by applying $20 \mathrm{kV}$ to the injection end of the column and approximately $2.0 \mathrm{kV}$ to the electrospray tip. To avoid adsorption, the capillary needed to be washed between analyses. The capillary was washed with $0.1 \mathrm{M} \mathrm{NaOH}$, followed by water and running buffer. After being rinsed, the capillary tube was equilibrated about 5 min before loading a sample.

All mass spectrometry experiments were conducted on an LCQ ion-trap mass spectrometer (Finnigan MAT, San Jose, CA). A commercial nanospray ESI source (Protana Co., Odense, Denmark) was used for CE/MS. This source assembly could be manipulated in $x, y$, and $z$ directions via micrometer screws. The carbon-coated capillary was inserted into a 32 gauge stainless steel needle for electrical contact (Figure 1). As shown in Figure 1, the carbon-coated fused-silica capillary was positioned at a distance of less than $2 \mathrm{~mm}$ from the entrance hole of the heated transfer capillary. The actual position was not found to be a critical factor. Nebulization gas was not necessary, and the heated transfer capillary was kept at a temperature of $200^{\circ} \mathrm{C}$. CE/ ESI-M S spectra were acquired in selected ion monitoring mode.

In the experiment comparing conventional sheath-flow and carbon-coated sheathless CE/ M S, the same capillary dimensions and buffer were used. A Cole-Palmer 100 syringe pump was used to deliver the sheath flow (50\%methanol containing $1 \%$ acetic acid) at a flow rate of $5 \mu \mathrm{L} / \mathrm{min}$. The CE buffer was $0.1 \%$ acetic acid (by

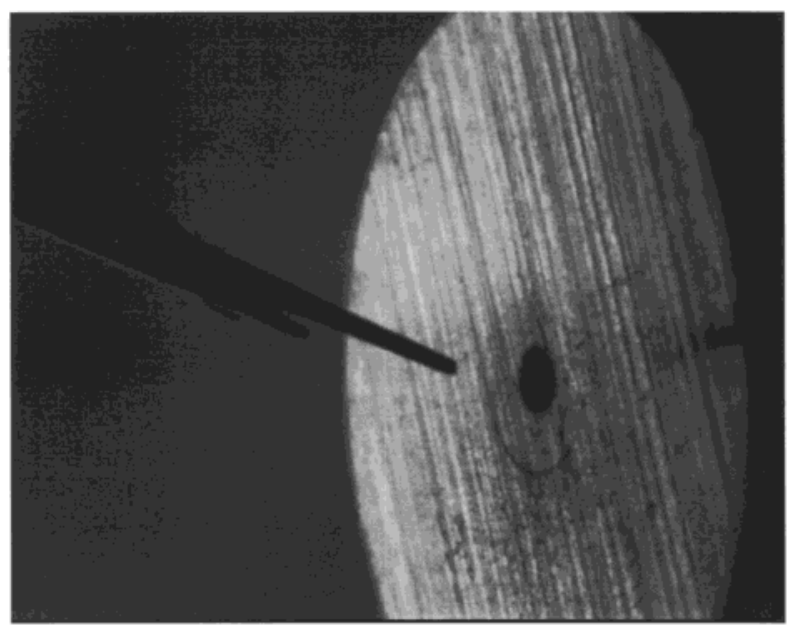

Figure 1. Photomicrograph of a tapered carbon-coated tip. The tip is positioned directly at the entrance hole of the heated transfer capillary.

volume). The sample was introduced hydrodynamically into the column ( $15 \mathrm{~cm} ; 10 \mathrm{~s})$. The injected volume was calculated to be $\sim 5.5 \mathrm{~nL}$.

In direct infusion experiments, the solution was supplied to the ESI emitter using a syringe pump with a gastight Hamilton syringe connected to the capillary through a TFL tube. The autotune mode was used for maximizing analyte ion signals in all ESI experiments. M ass spectra were obtained by averaging 20 scans, and each scan was built up from three microscans, requiring a maximum injection time of $200 \mathrm{~ms}$. For the carboncoated ESI emitter, the flow rate was set at $0.2 \mu \mathrm{L} / \mathrm{min}$ and the ESI voltage ranged from 1000 to $2500 \mathrm{~V}$. For the standard ESI emitter, the flow rate was set at $3.0 \mu \mathrm{L} / \mathrm{min}$ and the ESI voltage was $4500 \mathrm{~V}$.

\section{RESULTS AND DISCUSSION}

The Carbon-Coated Capillary. The carbon electrode is very popular in electrochemistry but is seldom used as the terminus of CE and ESI emitters. The properties of various types of carbon electrodes such as graphite, glassy carbon, and carbon paste make it the material of choice for many applications. In general, a carbon electrode has a low electrical resistance and a large useful potential window.

The procedure for making the carbon-coated capillary column is very simple. The tip of the fused-silica capillary is first smeared with a marker pen. This step is critical because it is quite difficult to coat the tip without the layer of marker pen ink. To make it easier to monitor the following carbon-coating process, a marker pen of black color should be avoided. After drying, the capillary tip is smeared with carbon using a soft pencil. It is noteworthy that it requires less than 1 min to prepare a carbon-coated fusedsilica capillary tip. Although it is rather simple to carbon-coat the tip, it does take some practice to make the tip. To avoid possible tip breakage, it is better to smear and coat the tip on the top of a soft cushion such as a computer mouse pad especially if the inner diameter of the tip is less than $15 \mu \mathrm{m}$.

A pencil is a hand-held device for writing, marking, or drawing; it consists of a core of solid marking substance that contains graphite, clay, and wax in a holder. The hardness and blackness 
(a)

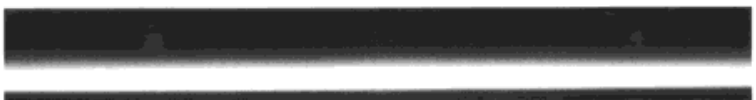

(b)

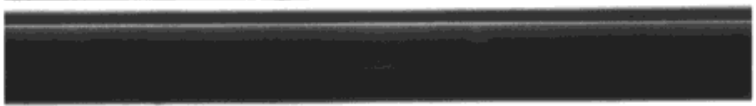

(c)
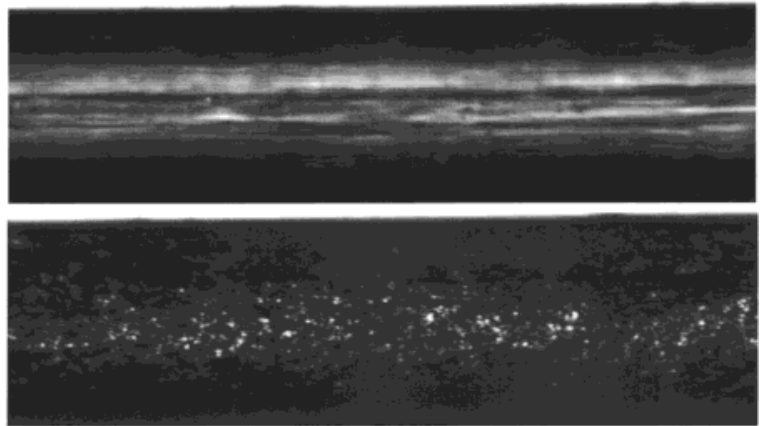

Figure 2. Microscopic graphs (100 $\times$ amplification) of the surface of fused-silica capillaries: (a) bare; (b) smeared with marker pen; (c) carbon-coated.

of a pencil are related to the proportions of clay (used as a binder) and graphite in the lead: the more graphite, the blacker and softer the pencil. The role of wax is to impart smoothness. Various types of pencils were investigated, and it was found that $4 B-6 B$ pencils produced the best interfaces. With harder pencils, such as $\mathrm{HB}$, it was difficult to smear the tip, and with soft pencils, such as $8 \mathrm{~B}$, it was difficult for the coating to adhere to the tip.

The thin layer of the marker pen ink provides the interface for the fused-silica surface and the carbon layer. Figure 2 shows optical microscopic graphs (100x amplification) of the fused-silica capillary surfaces. One possible explanation for the function of the marker pen layer is that the materials in the marker pen ink form a rough surface on the fused-silica capillary. This rough surface makes the coating of the carbon layer much easier.

As mentioned earlier, using a disposable gold-coated emitter is a very attractive approach for sheathlesS CE/ESI-MS. In comparison with the use of a disposable gold-coated emitter, this carbon coating approach has several advantages for sheathless CE/ ESI-M S applications. (1) Making the carbon-coated emitter by marker pen and pencil is easy, fast, and less expensive than the gold-coating method. (2) A one-piece separation column can offer higher separation efficiency than a butted column. (3) Although a gold-coated tip can be replaced, the performance of a butted column may not be very reproducible because of the different disposable emitter tips and new connection points between the tip and the column. One drawback of the carboncoating approach is that the lifetime of the tip is less than that of a gold-coated tip. This drawback, however, is not critical because the carbon-coated capillary can be easily refurbished.

Sheathless CE/ESI-MS. In sheathless CE/ ESI-M S, organic solvents such as methanol are often added to the CE buffer in order to improve its electrospray performance. To test the stability of the carbon-coated capillary tip, aqueous solutions of different methanol contents $(0-100 \%$ were examined. The coated capillary tips were immersed in the methanol/ water solutions for $30 \mathrm{~min}$. M icroscopic inspection (100x amplification) after the test revealed no perceptible coating deterioration in any methanol/ water solutions. Although the carbon layer remained intact, the layer of marker pen ink had dissolved in solution containing more than

Table 1. Stabilities in Different CE Solutions

\section{CE solutions ${ }^{a}$}

$1 \%$ acetic acid

$2 \%$ acetic acid

$3 \%$ acetic acid

4\%acetic acid

$3.125 \mathrm{mM}$ ammonium acetate

$6.25 \mathrm{mM}$ ammonium acetate

$12.5 \mathrm{mM}$ ammonium acetate

$25 \mathrm{mM}$ ammonium acetate acetate

$1 \%$ acetic acid $+3.125 \mathrm{mM}$ ammonium acetate

$2 \%$ acetic acid $+3.125 \mathrm{mM}$ ammonium acetate

$3 \%$ acetic acid +3.125 ammonium acetate
$50 \mathrm{mM}$ ammonium

$\begin{array}{cl}\begin{array}{c}\text { immersion and } \\ \text { sonication }\end{array} & \text { discharge } \\ \text { stable } & \text { no } \\ \text { stable } & \text { no } \\ \text { stable } & \text { yes } \\ \text { stable } & \text { yes } \\ \text { stable } & \text { no } \\ \text { stable } & \text { no } \\ \text { stable } & \text { yes/no }{ }^{d} \\ \text { stable } & \text { yes } \\ \text { stable } & \text { yes } \\ \text { stable } & \text { no } \\ \text { stable } & \text { yes } / \text { no }^{d} \\ \text { stable } & \text { yes }\end{array}$

a All of the solutions were in a $20 \%$ methanol/ water solution b Stability was based on visual inspection with a microscope. The coated capillary terminus was immersed in the solution and sonicated for 3 min. ${ }^{\mathrm{C}}$ Based on visual observation of glow in the dark. ${ }^{\mathrm{d}}$ Occasional discharge.

$60 \%$ methanol, as indicated by the color change of the solvents. Because there was no layer between the fused-silica surface and the carbon layer, the carbon-coated tip was found unstable. Perceptible coating deterioration was observed after sonication for $3 \mathrm{~min}$. In sheathless CE / ESI-M S applications, buffers containing up to $50 \%$ of methanol have been reported. ${ }^{12,17,19}$ No perceptible coating deterioration was observed for solutions containing less than $60 \%$ methanol.

It has been shown that, in comparison with the case of sheath liquid $\mathrm{CE} / \mathrm{MS}$, sensitivity can be improved by sheathless $\mathrm{CE} /$ M S. $13,17,20 \mathrm{~N}$ evertheless, the lack of sheath liquid restricts the use of high-concentration conductive buffers because these buffers can lead to corona discharge. Conductive reagents such as acetic acid or ammonium acetate solutions are often the choice for better separation performance. To test the useful range of these conductive buffers, various concentrations of acetic acid or ammonium acetate in a $20 \%$ methanol solution were examined. Table 1 summarizes the stabilities in different CE solutions. The results show that $20 \%$ methanol solutions containing less than $12.5 \mathrm{mM}$ ammonium acetate or $3 \%$ acetic acid were appropriate for sheathless CE/ ESI-M S using a carbon-coated capillary.

In CE/ ESI-M S, the carbon-coated tip acts as the electrode for both CE and ESI. The stability of the electrical contact at the CE terminus is a crucial factor for successful electrophoresis. The stability of the CE current was tested in a running buffer of $0.5 \%$ acetic acid (by volume) in a $20 \%$ methanol solution. The CE current was stable at $2.4 \pm 0.1 \mu \mathrm{A}$ for $70 \mathrm{~min}$, and the carboncoated column could be used for more than $8 \mathrm{~h}$ (maximum time studied).

Although the capillary tip with smaller inner diameter appears to have better sensitivity, other practical aspects such as the ease

(19) Cao, P.; M oini, M. Rapid Commun. M ass Spectrom. 1997, 8, 561-564.

(20) Gale, D. C.; Smith, R. D. Rapid Commun. Mass Spectrom. 1993, 7, 10171021. 


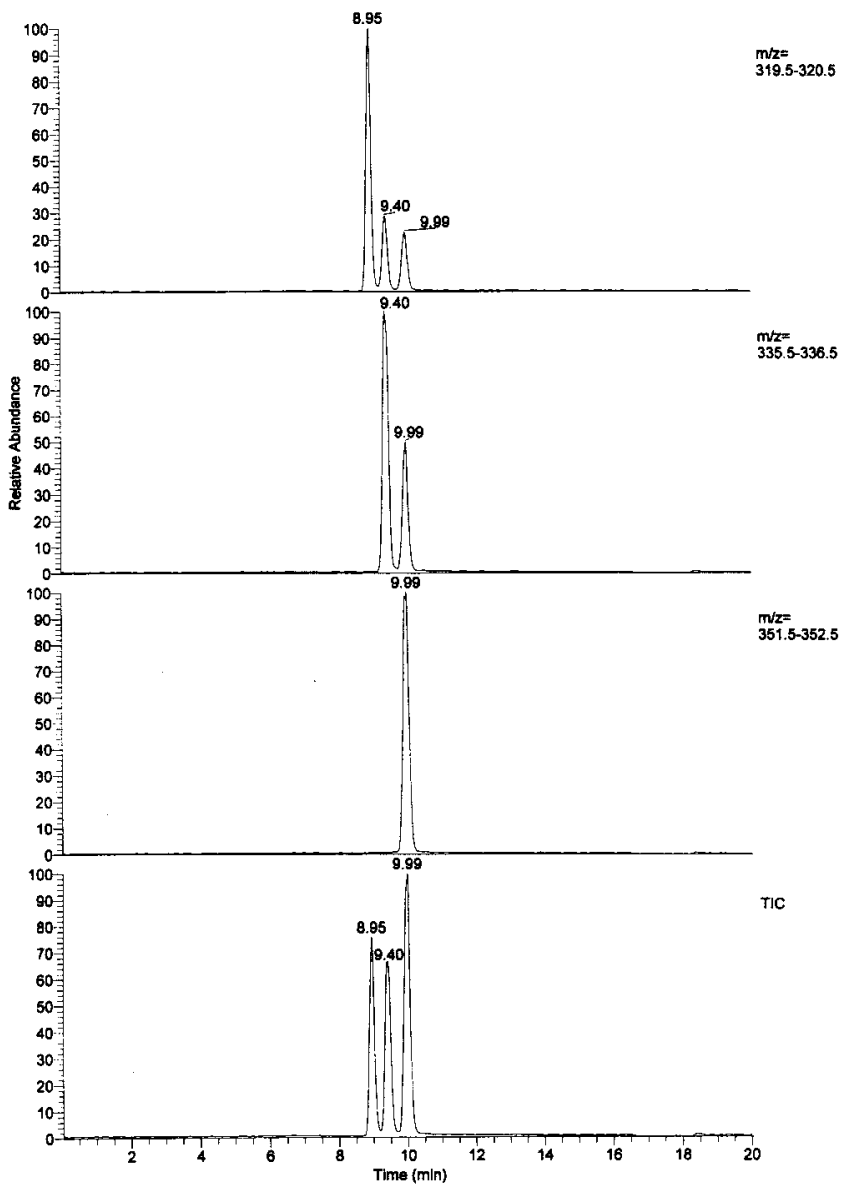

Figure 3. Sheathless CE/ES-MS electropherograms of coptisine, berberine, and palmatine. The CE buffer was $0.1 \%$ acetic acid (by volume) in $20 \%$ methanol solution; $\sim 7 \mathrm{pg}$ of each compound was injected hydrodynamically.

of production and occurrence of capillary blockages dictated the choice of capillary tip. In our experiment, a column tip tapered to less than $15 \mu \mathrm{m}$ i.d. was prone to blockage during the washing process. From a practical point of view, capillaries with a tip of $20 \mu \mathrm{m}$ i.d. provided the best compromise in terms of ease of use and sensitivity. Our result showed that the optimal flow rate for a $20 \mu \mathrm{m}$ i.d. tip was in the range $150-200 \mathrm{~nL} / \mathrm{min}$. To reach the optimal flow rate, a column with a $75 \mu \mathrm{m}$ i.d. was used for the analysis.

The usefulness of the carbon-coated tip in sheathless CE/ MS was demonstrated by the analysis of the three major bioactive components of Copti dis rhizoma (Huang-lien): coptisine chloride, berberine chloride, and palmatine chloride. Figure 3 shows the total ion electropherograms obtained from coptisine, berberine, and palmatine chlorides. All three compounds were resolved and detected in less than 10 min with peak widths of $30 \mathrm{~s}$ or less. The mass electropherograms at $\mathrm{m} / \mathrm{z} 320,336$, and 352 represent the molecular ions of coptisine, berberine, and palmatine. The small peaks in the traces of $\mathrm{m} / \mathrm{z} 320$ and $\mathrm{m} / \mathrm{z} 336$ were the fragment ions of berberine and palmatine, respectively.

The sensitivities of carbon-coated sheathless CE/MS and conventional sheath flow CE/ M S using the same capillary dimensions were compared using a mixture of coptisine, berberine, and
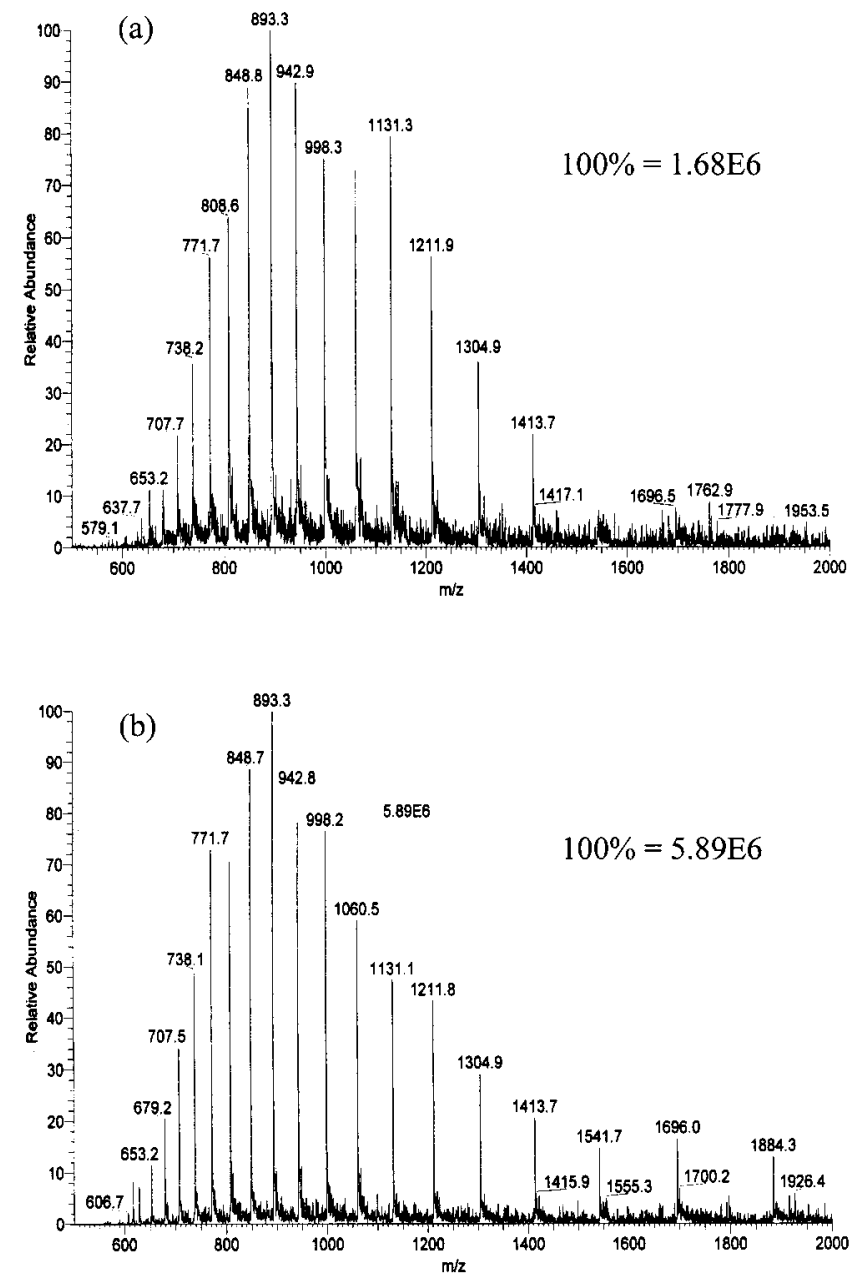

Figure 4. Mass spectra for $10 \mathrm{pmol} / \mu \mathrm{L}$ of horse heart myoglobin obtained with (a) standard electrospray and (b) microspray using a $25 \mu \mathrm{m}$ i.d. homemade carbon-coated capillary.

palmatine chlorides. The detection limit of sheathless CE/ M S is about $16 \mathrm{ppb}$, an enhancement in magnitude by a factor of $\sim 8$ in comparison with sheath flow CE/MS.

Microelectrospray. The carbon-coated capillary tip was also evaluated for the application of microelectrospray. The durability of the carbon coating under electrospray was estimated as follows. A $10 \mathrm{mM}$ solution of acetic acid in water (pH 3.44) was sprayed at $0.2 \mu \mathrm{L} / \mathrm{min}$ from a $25 \mu \mathrm{m}$ i.d. carbon-coated tip. There was no noticeable change in the carbon-coated emitter after operating for $30 \mathrm{~h}$ (maximum time studied).

Microelectrospray was evaluated by infusing horse heart myoglobin into a $0.1 \%$ (by volume) formic acid/ $50 \%$ acetonitrile solution. Spectra were obtained by averaging 20 scans, and the heated transfer capillary was kept at a temperature of $100{ }^{\circ} \mathrm{C}$. Figure 4 shows the results obtained for the horse heart myoglobin at $10 \mathrm{pmol} / \mu \mathrm{L}$ using standard electrospray (Figure 4a) and using microspray with a $25 \mu \mathrm{m}$ i.d. homemade carbon-coated capillary (Figure $4 \mathrm{~b}$ ). The spectra are similar, with multiply charged molecular ions ranging from the $9+$ to $29+$. The total amount of sample consumed in microspray was over 1 order of magnitude less than that of the standard ESI setup, while the intensity of the base peak was about 3.5 times greater. 


\section{CONCLUSIONS}

A carbon-coated capillary has been shown to be useful for microspray and sheathless CE/ ESI-M S applications. The carboncoating process is simple and less expensive than the preparation of gold-coated capillaries. M oreover, the carbon-coated tip can be fabricated or refurbished in less than $1 \mathrm{~min}$. The mechanism of carbon coating is not well understood. The marker pen ink may function as an adhesive for the graphitized material or it may simply provide a rough surface so that graphitized material can be retained on the rough surface. Work is in progress to investigate the function of the marker pen layer in the carbon- coating process. It is hoped that, with more knowledge about the function of the marker pen layer, a better interface can be developed.

\section{ACKNOWLEDGMENT}

We thank the National Science Council of the Republic of China for financial support.

Received for review May 18, 1999. Accepted October 19, 1999.

AC990535E 